\title{
Theoretical Study on the Selectivity of Asymmetric Sulfur Ylide Epoxidation Reaction
}

\author{
María A. Silva, Benjamin R. Bellenie and Jonathan M. Goodman* \\ Unilever Centre for Molecular Science Informatics, Department of Chemistry, University of Cambridge, Lensfield \\ Road, Cambridge, CB2 1EW, U. K.
}

Supplementary Information. List of contents:

- Cartesian coordinates and absolute energies (B3LYP/6-31G*, in hartrees), and number of imaginary frequencies of all stationary points reported in the paper; values of imaginary frequencies of all transition structures. Pages S1-S7.

- Single point energies in acetonitrile (at B3LYP/6-31G*, in hartrees) for the transition structures for the addition reaction of sulfonium ylide 2 to benzaldehyde.

- Plots of the relative energy (in kcal mol ${ }^{-1}$ ) versus mass-weighted coordinates (in bohr amu ${ }^{1 / 2}$ ), from B3LYP/6-31G* quick reaction coordinate (QRC) calculations, for the transition structures TS2s (Figure S1). Pages S8-S9.

- Transition structures connecting betaines with epoxides for the trans pathway and with the chiral sulfide 2 (Figure S2) (B3LYP/6-31G* optimized geometries and energies in gas phase and single point energies in acetonitrile) and QRC calculations (at B3LYP/6-31G*) for these TSs (Figure S3). Pages S10-S11.

\begin{tabular}{|c|c|c|c|}
\hline \multicolumn{4}{|c|}{$\begin{array}{l}\text { Sulfonium Ylide 1-in } \\
\text { B3LYP/6-31G* GEOMETRY }\end{array}$} \\
\hline C & 0.126855 & -0.375447 & 0.854029 \\
\hline C & 1.057842 & -1.491807 & 0.844818 \\
\hline C & 2.233576 & -1.570755 & 0.066011 \\
\hline C & 3.039006 & -2.709141 & 0.088064 \\
\hline C & 2.712309 & -3.805894 & 0.888114 \\
\hline C & 1.559753 & -3.740605 & 1.679022 \\
\hline C & 0.749870 & -2.609585 & 1.658569 \\
\hline C & 1.353668 & 2.342449 & -0.161453 \\
\hline C & 0.978344 & 1.755803 & -2.546819 \\
\hline C & 1.998590 & 2.319251 & -1.546943 \\
\hline $\mathrm{O}$ & 0.023490 & 2.792119 & -2.766795 \\
\hline $\mathrm{O}$ & 3.129241 & 1.455936 & -1.597913 \\
\hline C & 2.341900 & 2.243516 & 0.993122 \\
\hline $\mathrm{O}$ & 3.448993 & 1.397425 & 0.704080 \\
\hline C & 4.040070 & 1.699881 & -0.530000 \\
\hline C & 5.270080 & 0.845303 & -0.741952 \\
\hline $\mathrm{C}$ & 5.822997 & 0.739826 & -2.024625 \\
\hline C & 6.976212 & -0.017341 & -2.232139 \\
\hline C & 7.586179 & -0.673771 & -1.159353 \\
\hline C & 7.035961 & -0.569275 & 0.119850 \\
\hline C & 5.881418 & 0.188673 & 0.330867 \\
\hline C & -0.995004 & 0.180568 & -2.652362 \\
\hline $\mathrm{O}$ & -1.800869 & 1.335932 & -2.850374 \\
\hline C & -1.094939 & 2.348490 & -3.524953 \\
\hline C & -2.006519 & 3.527037 & -3.783767 \\
\hline C & -1.455130 & 4.784205 & -4.059295 \\
\hline C & -2.286406 & 5.867643 & -4.344763 \\
\hline C & -3.673980 & 5.702725 & -4.358848 \\
\hline C & -4.224977 & 4.449134 & -4.085403 \\
\hline C & -3.395415 & 3.362685 & -3.800343 \\
\hline$S$ & -0.036676 & 0.959219 & -0.132664 \\
\hline $\mathrm{C}$ & 0.307488 & 0.527024 & -1.938154 \\
\hline $\mathrm{H}$ & -0.692959 & -0.402759 & 1.563721 \\
\hline $\mathrm{H}$ & 2.548062 & -0.723879 & -0.530981 \\
\hline $\mathrm{H}$ & 3.940947 & -2.725819 & -0.519554 \\
\hline $\mathrm{H}$ & 3.344175 & -4.689985 & 0.902222 \\
\hline $\mathrm{H}$ & 1.287706 & -4.579700 & 2.315828 \\
\hline $\mathrm{H}$ & -0.147254 & -2.580678 & 2.273965 \\
\hline $\mathrm{H}$ & 1.487421 & 1.493566 & -3.487517 \\
\hline $\mathrm{H}$ & 0.748655 & 3.247141 & -0.056481 \\
\hline $\mathrm{H}$ & 2.288273 & 3.334998 & -1.860841 \\
\hline
\end{tabular}

\begin{tabular}{|c|c|c|c|}
\hline & \\
\hline $\mathrm{H}$ & 2.707470 & 3.252332 & 1.247891 \\
\hline $\mathrm{H}$ & 1.859122 & 1.812967 & 1.877747 \\
\hline $\mathrm{H}$ & 4.299619 & 2.777192 & -0.553242 \\
\hline $\mathrm{H}$ & 5.338784 & 1.238788 & -2.858802 \\
\hline $\mathrm{H}$ & 7.395397 & -0.099122 & -3.231780 \\
\hline $\mathrm{H}$ & 8.483621 & -1.265798 & -1.321224 \\
\hline $\mathrm{H}$ & 7.501213 & -1.082903 & 0.957187 \\
\hline $\mathrm{H}$ & 5.442777 & 0.261932 & 1.319679 \\
\hline $\mathrm{H}$ & -0.760572 & -0.286879 & -3.623804 \\
\hline $\mathrm{H}$ & -1.590970 & -0.524346 & -2.066210 \\
\hline $\mathrm{H}$ & -0.708657 & 1.932369 & -4.476583 \\
\hline $\mathrm{H}$ & -0.377371 & 4.911577 & -4.037064 \\
\hline $\mathrm{H}$ & -1.850305 & 6.841196 & -4.553230 \\
\hline $\mathrm{H}$ & -4.321463 & 6.547141 & -4.580713 \\
\hline $\mathrm{H}$ & -5.303553 & 4.314693 & -4.090844 \\
\hline $\mathrm{H}$ & -3.819123 & 2.388820 & -3.580791 \\
\hline $\mathrm{H}$ & 0.983777 & -0.330031 & -1.938371 \\
\hline \multicolumn{4}{|c|}{ B3LYP/6-31G* Energy $=-1743.50857742416$} \\
\hline \multicolumn{4}{|c|}{ Number of Imaginary Frequencies $=0$} \\
\hline \multicolumn{4}{|c|}{ Sulfonium Ylide 1-out } \\
\hline \multicolumn{4}{|c|}{ B3LYP/6-31G* GEOMETRY } \\
\hline $\mathrm{C}$ & 0.373054 & -0.197135 & 0.930753 \\
\hline $\mathrm{C}$ & -0.390168 & -0.581036 & 2.109322 \\
\hline $\mathrm{C}$ & -1.637502 & -0.032560 & 2.487344 \\
\hline $\mathrm{C}$ & -2.307448 & -0.478962 & 3.622987 \\
\hline $\mathrm{C}$ & -1.769525 & -1.488876 & 4.428150 \\
\hline $\mathrm{C}$ & -0.538875 & -2.044533 & 4.068729 \\
\hline $\mathrm{C}$ & 0.138565 & -1.599873 & 2.935951 \\
\hline $\mathrm{C}$ & 1.291353 & 2.495103 & -0.187523 \\
\hline $\mathrm{C}$ & 0.953082 & 1.742439 & -2.518119 \\
\hline $\mathrm{C}$ & 1.983690 & 2.315464 & -1.537744 \\
\hline $\mathrm{O}$ & 0.013661 & 2.778791 & -2.785790 \\
\hline $\mathrm{O}$ & 3.021287 & 1.341220 & -1.477911 \\
\hline $\mathrm{C}$ & 2.246019 & 2.445576 & 0.996002 \\
\hline $\mathrm{O}$ & 3.232062 & 1.430874 & 0.838022 \\
\hline $\mathrm{C}$ & 3.910332 & 1.535626 & -0.384689 \\
\hline $\mathrm{C}$ & 4.994641 & 0.481975 & -0.459207 \\
\hline $\mathrm{C}$ & 5.553963 & 0.149192 & -1.699342 \\
\hline $\mathrm{C}$ & 6.570703 & -0.802938 & -1.778348 \\
\hline $\mathrm{C}$ & 7.037028 & -1.429146 & -0.619121 \\
\hline $\mathrm{C}$ & 6.481925 & -1.096818 & 0.618219 \\
\hline $\mathrm{C}$ & 5.465036 & -0.142704 & 0.700569 \\
\hline
\end{tabular}




$\begin{array}{lrrr}\mathrm{C} & -1.031457 & 0.182963 & -2.527042 \\ \mathrm{O} & -1.826818 & 1.336879 & -2.773253 \\ \mathrm{C} & -1.121173 & 2.310008 & -3.505356 \\ \mathrm{C} & -2.026808 & 3.483918 & -3.802149 \\ \mathrm{C} & -1.470529 & 4.729038 & -4.119369 \\ \mathrm{C} & -2.298098 & 5.806478 & -4.436943 \\ \mathrm{C} & -3.686407 & 5.647436 & -4.442009 \\ \mathrm{C} & -4.242229 & 4.405610 & -4.127289 \\ \mathrm{C} & -3.416582 & 3.325141 & -3.809828 \\ \mathrm{~S} & -0.051547 & 1.081156 & -0.052747 \\ \mathrm{C} & 0.273088 & 0.559844 & -1.834242 \\ \mathrm{H} & 1.345812 & -0.650696 & 0.770683 \\ \mathrm{H} & -2.086842 & 0.751182 & 1.881949 \\ \mathrm{H} & -3.264951 & -0.031509 & 3.881964 \\ \mathrm{H} & -2.297413 & -1.832972 & 5.313457 \\ \mathrm{H} & -0.097142 & -2.831316 & 4.676975 \\ \mathrm{H} & 1.097470 & -2.043117 & 2.674792 \\ \mathrm{H} & 1.451959 & 1.420290 & -3.445479 \\ \mathrm{H} & 0.706392 & 3.418775 & -0.182452 \\ \mathrm{H} & 2.377748 & 3.273474 & -1.913676 \\ \mathrm{H} & 2.734630 & 3.426458 & 1.121314 \\ \mathrm{H} & 1.713309 & 2.199128 & 1.919800 \\ \mathrm{H} & 4.337408 & 2.554120 & -0.473245 \\ \mathrm{H} & 5.180172 & 0.626185 & -2.600241 \\ \mathrm{H} & 6.995424 & -1.059458 & -2.745512 \\ \mathrm{H} & 7.827031 & -2.173292 & -0.681192 \\ \mathrm{H} & 6.836577 & -1.583181 & 1.523286 \\ \mathrm{H} & 5.025334 & 0.112057 & 1.658572 \\ \mathrm{H} & -0.803942 & -0.329461 & -3.477159 \\ \mathrm{H} & -1.631687 & -0.489427 & -1.907921 \\ \mathrm{H} & -0.753759 & 1.845719 & -4.441835 \\ \mathrm{H} & -0.392237 & 4.852303 & -4.103978 \\ \mathrm{H} & -1.858338 & 6.771074 & -4.677044 \\ \mathrm{H} & -4.330833 & 6.487404 & -4.688474 \\ \mathrm{H} & -5.321387 & 4.276005 & -4.125099 \\ \mathrm{H} & -3.844371 & 2.361090 & -3.557015 \\ \mathrm{H} & 0.951855 & -0.294683 & -1.768576\end{array}$

B3LYP/6-31G* Energy $=-1743.50981752449$

Number of Imaginary Frequencies $=0$

Sulfonium Ylide 2-in

B3LYP/6-31G* GEOMETRY

$\begin{array}{lrrr}\mathrm{C} & 0.615370 & -1.017365 & 1.073771 \\ \mathrm{C} & 0.684377 & -1.873196 & -0.090326 \\ \mathrm{C} & 0.685235 & -1.432221 & -1.435455 \\ \mathrm{C} & 0.731804 & -2.331922 & -2.500079 \\ \mathrm{C} & 0.787947 & -3.708174 & -2.275151 \\ \mathrm{C} & 0.798571 & -4.167950 & -0.952794 \\ \mathrm{C} & 0.746261 & -3.276386 & 0.112133 \\ \mathrm{H} & 0.694811 & -1.468910 & 2.055869 \\ \mathrm{H} & 0.660643 & -0.369675 & -1.656229 \\ \mathrm{H} & 0.729142 & -1.947093 & -3.517995 \\ \mathrm{H} & 0.826339 & -4.406228 & -3.106800 \\ \mathrm{H} & 0.845523 & -5.235871 & -0.749626 \\ \mathrm{H} & 0.748813 & -3.657152 & 1.131404 \\ \mathrm{C} & 1.625825 & 1.809281 & 0.321099 \\ \mathrm{C} & -0.601202 & 2.565855 & -0.474220 \\ \mathrm{C} & 0.883743 & 2.432586 & -0.860703 \\ \mathrm{H} & -1.216820 & 2.847873 & -1.337105 \\ \mathrm{H} & -0.721528 & 3.356538 & 0.279974 \\ \mathrm{H} & 0.982321 & 1.790653 & -1.745164 \\ \mathrm{H} & 1.787878 & 2.571199 & 1.093687 \\ \mathrm{H} & 1.314796 & 3.407048 & -1.122811 \\ \mathrm{~S} & 0.359315 & 0.632952 & 1.202837 \\ \mathrm{C} & -1.067244 & 1.229186 & 0.100520 \\ \mathrm{H} & -1.144767 & 0.478216 & -0.691265 \\ \mathrm{C} & 2.929837 & 1.089406 & 0.019508 \\ \mathrm{H} & 2.795562 & 0.320609 & -0.746421 \\ \mathrm{H} & 3.679727 & 1.807484 & -0.335190 \\ \mathrm{H} & 3.326539 & 0.598471 & 0.914373 \\ \mathrm{C} & -2.353773 & 1.261513 & 0.915164 \\ \mathrm{H} & -2.563083 & 0.278438 & 1.350304 \\ \mathrm{H} & -2.293370 & 1.993068 & 1.729442 \\ \mathrm{H} & -3.201210 & 1.532553 & 0.273478 \\ & & & \end{array}$

B3LYP/6-31G* Energy $=-904.34865666721$
Number of Imaginary Frequencies $=0$

Sulfonium Ylide 2-out

B3LYP/6-31G* GEOMETRY

$\begin{array}{rrrr}\mathrm{C} & 0.486015 & -1.017590 & 0.971476 \\ \mathrm{C} & 0.803061 & -1.993263 & 2.000568 \\ \mathrm{C} & 0.892297 & -1.712622 & 3.384814 \\ \mathrm{C} & 1.165167 & -2.715317 & 4.310677 \\ \mathrm{C} & 1.369791 & -4.037541 & 3.903785 \\ \mathrm{C} & 1.293261 & -4.333750 & 2.540483 \\ \mathrm{C} & 1.018262 & -3.336166 & 1.608938 \\ \mathrm{H} & 0.529247 & -1.326824 & -0.069738 \\ \mathrm{H} & 0.744375 & -0.694758 & 3.737377 \\ \mathrm{H} & 1.223499 & -2.457604 & 5.366545 \\ \mathrm{H} & 1.587339 & -4.815280 & 4.630702 \\ \mathrm{H} & 1.450558 & -5.353641 & 2.195016 \\ \mathrm{H} & 0.961931 & -3.590609 & 0.552252 \\ \mathrm{C} & 1.600703 & 1.811795 & 0.471902 \\ \mathrm{C} & -0.565274 & 2.532170 & -0.476086 \\ \mathrm{C} & 0.931612 & 2.361745 & -0.785041 \\ \mathrm{H} & -1.141077 & 2.790730 & -1.372892 \\ \mathrm{H} & -0.710885 & 3.345695 & 0.248213 \\ \mathrm{H} & 1.058395 & 1.649931 & -1.612801 \\ \mathrm{H} & 1.702399 & 2.602648 & 1.224835 \\ \mathrm{H} & 1.389742 & 3.307861 & -1.099635 \\ \mathrm{~S} & 0.282050 & 0.615152 & 1.286799 \\ \mathrm{C} & -1.063989 & 1.213537 & 0.110826 \\ \mathrm{H} & -1.081281 & 0.440385 & -0.667118 \\ \mathrm{C} & 2.912097 & 1.074154 & 0.276220 \\ \mathrm{H} & 2.772569 & 0.187199 & -0.352078 \\ \mathrm{H} & 3.648042 & 1.727688 & -0.208079 \\ \mathrm{H} & 3.331110 & 0.736951 & 1.229986 \\ \mathrm{C} & -2.406306 & 1.257277 & 0.830039 \\ \mathrm{H} & -2.642319 & 0.282400 & 1.269202 \\ \mathrm{H} & -2.405351 & 2.006079 & 1.630237 \\ \mathrm{H} & -3.204684 & 1.513537 & 0.123183 \\ & & & \end{array}$

B3LYP/6-31G* Energy = -904.34830634337

Number of Imaginary Frequencies $=0$

Benzaldehyde

B3LYP/6-31G* GEOMETRY

$\begin{array}{lrrl}\mathrm{C} & -0.900965 & 1.795880 & 0.000000 \\ \mathrm{O} & -2.113427 & 1.890829 & 0.000000 \\ \mathrm{H} & -0.260919 & 2.706439 & 0.000000 \\ \mathrm{C} & 1.282802 & -1.874775 & 0.000000 \\ \mathrm{C} & 1.967512 & -0.656599 & 0.000000 \\ \mathrm{C} & 1.249457 & 0.538543 & 0.000000 \\ \mathrm{C} & -0.151610 & 0.518722 & 0.000000 \\ \mathrm{C} & -0.834915 & -0.707407 & 0.000000 \\ \mathrm{C} & -0.117868 & -1.899708 & 0.000000 \\ \mathrm{H} & 1.840640 & -2.807754 & 0.000000 \\ \mathrm{H} & 3.053957 & -0.640762 & 0.000000 \\ \mathrm{H} & 1.771365 & 1.493767 & 0.000000 \\ \mathrm{H} & -1.921027 & -0.698128 & 0.000000 \\ \mathrm{H} & -0.642928 & -2.851199 & 0.000000\end{array}$

B3LYP/6-31G* Energy $=-345.57328151886$ Number of Imaginary Frequencies $=0$ 
Transition Structures for the Addition Reaction of Sulfonium Ylide 1 to Benzaldehyde.

TS1-RS

B3LYP/6-31G* GEOMETRY

\begin{tabular}{|c|c|c|c|}
\hline $\mathrm{C}$ & 0.795655 & -1.742394 & -0.950968 \\
\hline $\mathrm{C}$ & 0.835986 & -2.435359 & 0.357172 \\
\hline $\mathrm{C}$ & 1.922446 & -2.244782 & 1.242220 \\
\hline $\mathrm{C}$ & 2.021512 & -2.976178 & 2.426757 \\
\hline $\mathrm{C}$ & 1.061999 & -3.941467 & 2.743394 \\
\hline $\mathrm{C}$ & 0.002397 & -4.169595 & 1.860462 \\
\hline $\mathrm{C}$ & -0.114552 & -3.420934 & 0.689502 \\
\hline $\mathrm{H}$ & 0.087510 & -2.183063 & -1.657238 \\
\hline $\mathrm{H}$ & 2.711041 & -1.549755 & 0.963691 \\
\hline $\mathrm{H}$ & 2.864180 & -2.805852 & 3.092993 \\
\hline $\mathrm{H}$ & 1.143831 & -4.516152 & 3.662548 \\
\hline $\mathrm{H}$ & -0.745957 & -4.923524 & 2.092382 \\
\hline $\mathrm{H}$ & -0.956709 & -3.588314 & 0.024593 \\
\hline $\mathrm{C}$ & 2.711985 & -1.645262 & -1.747718 \\
\hline $\mathrm{O}$ & 3.218050 & -0.544477 & -1.376787 \\
\hline $\mathrm{C}$ & 2.418099 & -1.889503 & -3.209715 \\
\hline $\mathrm{C}$ & 2.441994 & -0.823945 & -4.118253 \\
\hline $\mathrm{C}$ & 2.202300 & -1.047818 & -5.476282 \\
\hline $\mathrm{C}$ & 1.953792 & -2.342040 & -5.942969 \\
\hline $\mathrm{C}$ & 1.939490 & -3.413356 & -5.042184 \\
\hline $\mathrm{C}$ & 2.166699 & -3.184680 & -3.685101 \\
\hline $\mathrm{H}$ & 2.994008 & -2.581970 & -1.225699 \\
\hline $\mathrm{H}$ & 2.672751 & 0.166549 & -3.736769 \\
\hline $\mathrm{H}$ & 2.222922 & -0.214582 & -6.175491 \\
\hline $\mathrm{H}$ & 1.783771 & -2.518645 & -7.002605 \\
\hline $\mathrm{H}$ & 1.760400 & -4.424571 & -5.401041 \\
\hline $\mathrm{H}$ & 2.161123 & -4.016783 & -2.982116 \\
\hline $\mathrm{C}$ & -1.338184 & 0.515536 & -0.902320 \\
\hline $\mathrm{C}$ & -0.236811 & 1.711012 & 0.989188 \\
\hline $\mathrm{C}$ & -1.547977 & 1.101921 & 0.507357 \\
\hline $\mathrm{H}$ & -0.248585 & 1.781374 & 2.088332 \\
\hline $\mathrm{O}$ & -0.154153 & 3.004506 & 0.416213 \\
\hline $\mathrm{O}$ & -1.910904 & 0.105861 & 1.445944 \\
\hline $\mathrm{H}$ & -1.431440 & 1.318711 & -1.639960 \\
\hline $\mathrm{H}$ & -2.321357 & 1.885321 & 0.466229 \\
\hline $\mathrm{C}$ & -2.356282 & -0.587579 & -1.235252 \\
\hline $\mathrm{H}$ & -3.275409 & -0.096835 & -1.594831 \\
\hline $\mathrm{H}$ & -1.994699 & -1.248007 & -2.027338 \\
\hline $\mathrm{O}$ & -2.663574 & -1.419732 & -0.125514 \\
\hline $\mathrm{C}$ & -3.022943 & -0.649304 & 0.998214 \\
\hline $\mathrm{H}$ & -3.813463 & 0.064065 & 0.689148 \\
\hline $\mathrm{C}$ & -3.518013 & -1.511212 & 2.134759 \\
\hline $\mathrm{C}$ & -3.554645 & -0.967158 & 3.425413 \\
\hline $\mathrm{C}$ & -4.060752 & -1.713827 & 4.488429 \\
\hline $\mathrm{C}$ & -4.535935 & -3.009670 & 4.269247 \\
\hline $\mathrm{C}$ & -4.506948 & -3.550074 & 2.981744 \\
\hline $\mathrm{C}$ & -4.003397 & -2.802722 & 1.913691 \\
\hline $\mathrm{H}$ & -3.175602 & 0.036043 & 3.593665 \\
\hline $\mathrm{H}$ & -4.079894 & -1.284890 & 5.487056 \\
\hline $\mathrm{H}$ & -4.925944 & -3.593910 & 5.098699 \\
\hline $\mathrm{H}$ & -4.875414 & -4.557319 & 2.806630 \\
\hline $\mathrm{H}$ & -3.979088 & -3.218970 & 0.912128 \\
\hline $\mathrm{C}$ & 2.274402 & 1.580282 & 0.531269 \\
\hline $\mathrm{H}$ & 2.649376 & 1.644851 & 1.566182 \\
\hline $\mathrm{H}$ & 2.996370 & 1.049010 & -0.090681 \\
\hline $\mathrm{O}$ & 2.121108 & 2.893769 & -0.001654 \\
\hline $\mathrm{C}$ & 1.116490 & 3.599302 & 0.670498 \\
\hline $\mathrm{H}$ & 1.299233 & 3.527837 & 1.761688 \\
\hline $\mathrm{C}$ & 1.074457 & 5.048184 & 0.246797 \\
\hline $\mathrm{C}$ & -0.067822 & 5.810885 & 0.523083 \\
\hline $\mathrm{C}$ & -0.101039 & 7.167361 & 0.200763 \\
\hline $\mathrm{C}$ & 1.008620 & 7.772142 & -0.396394 \\
\hline $\mathrm{C}$ & 2.150077 & 7.013952 & -0.665972 \\
\hline $\mathrm{C}$ & 2.186437 & 5.654992 & -0.343903 \\
\hline $\mathrm{H}$ & -0.929429 & 5.336829 & 0.983483 \\
\hline $\mathrm{H}$ & -0.992700 & 7.751215 & 0.414990 \\
\hline $\mathrm{H}$ & 0.982542 & 8.829092 & -0.649006 \\
\hline $\mathrm{H}$ & 3.016019 & 7.479567 & -1.128982 \\
\hline $\mathrm{H}$ & 3.069443 & 5.059953 & -0.551060 \\
\hline $\mathrm{S}$ & 0.486399 & 0.046642 & -1.067675 \\
\hline $\mathrm{C}$ & 0.943487 & 0.811197 & 0.570811 \\
\hline $\mathrm{H}$ & 1.016433 & -0.031775 & 1.262391 \\
\hline
\end{tabular}

B3LYP/6-31G* Energy $=-2089.05615413414$

Number of Imaginary Frequencies $=1$

Imaginary Frequency $=-232.78$

TS1-SR

B3LYP/6-31G* GEOMETRY

\begin{tabular}{|c|c|c|c|}
\hline $\mathrm{C}$ & 0.075198 & -1.865471 & 0.235082 \\
\hline $\mathrm{C}$ & 1.209534 & -2.795348 & 0.364780 \\
\hline $\mathrm{C}$ & 1.700779 & -3.227025 & 1.616488 \\
\hline $\mathrm{C}$ & 2.715639 & -4.181018 & 1.697301 \\
\hline $\mathrm{C}$ & 3.242831 & -4.757984 & 0.538028 \\
\hline $\mathrm{C}$ & 2.743898 & -4.363419 & -0.707329 \\
\hline $\mathrm{C}$ & 1.752146 & -3.387893 & -0.794789 \\
\hline $\mathrm{H}$ & -0.079359 & -1.512248 & -0.785024 \\
\hline $\mathrm{H}$ & 1.275849 & -2.816535 & 2.530267 \\
\hline $\mathrm{H}$ & 3.085679 & -4.484831 & 2.673636 \\
\hline $\mathrm{H}$ & 4.028586 & -5.505614 & 0.604232 \\
\hline $\mathrm{H}$ & 3.140337 & -4.805701 & -1.618370 \\
\hline $\mathrm{H}$ & 1.391016 & -3.068103 & -1.769067 \\
\hline $\mathrm{C}$ & -1.578439 & -2.702196 & 1.090320 \\
\hline $\mathrm{O}$ & -1.980051 & -1.891096 & 1.987249 \\
\hline $\mathrm{C}$ & -2.481758 & -3.046172 & -0.077121 \\
\hline $\mathrm{C}$ & -2.084179 & -3.952086 & -1.072211 \\
\hline $\mathrm{C}$ & -2.958733 & -4.311450 & -2.097512 \\
\hline $\mathrm{C}$ & -4.254227 & -3.782744 & -2.133346 \\
\hline $\mathrm{C}$ & -4.663799 & -2.893024 & -1.137081 \\
\hline $\mathrm{C}$ & -3.781534 & -2.527018 & -0.117605 \\
\hline $\mathrm{H}$ & -0.990595 & -3.587544 & 1.405374 \\
\hline $\mathrm{H}$ & -1.084175 & -4.379360 & -1.036568 \\
\hline $\mathrm{H}$ & -2.636372 & -5.011616 & -2.864960 \\
\hline $\mathrm{H}$ & -4.941443 & -4.073675 & -2.924442 \\
\hline $\mathrm{H}$ & -5.676959 & -2.496546 & -1.145666 \\
\hline $\mathrm{H}$ & -4.094773 & -1.866404 & 0.685403 \\
\hline $\mathrm{C}$ & 1.566296 & 0.693010 & 0.931373 \\
\hline $\mathrm{C}$ & -0.419372 & 2.047803 & 0.282537 \\
\hline $\mathrm{C}$ & 1.051348 & 1.795894 & -0.014740 \\
\hline $\mathrm{H}$ & -0.894112 & 2.542838 & -0.578977 \\
\hline $\mathrm{O}$ & -0.451044 & 2.895459 & 1.419629 \\
\hline $\mathrm{O}$ & 1.149101 & 1.413964 & -1.375653 \\
\hline $\mathrm{H}$ & 1.794685 & 1.142526 & 1.903066 \\
\hline $\mathrm{H}$ & 1.623150 & 2.720713 & 0.161169 \\
\hline $\mathrm{C}$ & 2.840040 & 0.025224 & 0.380366 \\
\hline $\mathrm{H}$ & 3.686191 & 0.688935 & 0.623722 \\
\hline $\mathrm{H}$ & 3.023962 & -0.949640 & 0.831232 \\
\hline $\mathrm{O}$ & 2.790042 & -0.179346 & -1.025377 \\
\hline $\mathrm{C}$ & 2.475091 & 1.014042 & -1.695248 \\
\hline $\mathrm{H}$ & 3.158231 & 1.809510 & -1.335454 \\
\hline $\mathrm{C}$ & 2.600933 & 0.869030 & -3.192043 \\
\hline $\mathrm{C}$ & 2.031451 & 1.851995 & -4.012392 \\
\hline $\mathrm{C}$ & 2.192711 & 1.791758 & -5.395746 \\
\hline $\mathrm{C}$ & 2.926902 & 0.749414 & -5.969103 \\
\hline $\mathrm{C}$ & 3.497217 & -0.228955 & -5.151754 \\
\hline $\mathrm{C}$ & 3.339082 & -0.170310 & -3.764204 \\
\hline $\mathrm{H}$ & 1.458252 & 2.657320 & -3.562865 \\
\hline $\mathrm{H}$ & 1.745793 & 2.557469 & -6.024658 \\
\hline $\mathrm{H}$ & 3.053266 & 0.702242 & -7.047655 \\
\hline $\mathrm{H}$ & 4.068357 & -1.041008 & -5.593725 \\
\hline $\mathrm{H}$ & 3.780926 & -0.926588 & -3.123988 \\
\hline $\mathrm{C}$ & -2.436468 & 0.914390 & 1.343949 \\
\hline $\mathrm{H}$ & -3.202023 & 1.267157 & 0.632267 \\
\hline $\mathrm{H}$ & -2.742837 & -0.034959 & 1.784324 \\
\hline $\mathrm{O}$ & -2.283294 & 1.867041 & 2.390081 \\
\hline $\mathrm{C}$ & -1.780052 & 3.077610 & 1.901146 \\
\hline $\mathrm{H}$ & -2.398966 & 3.399684 & 1.039632 \\
\hline $\mathrm{C}$ & -1.781554 & 4.146278 & 2.967915 \\
\hline $\mathrm{C}$ & -0.979668 & 5.283366 & 2.807874 \\
\hline $\mathrm{C}$ & -1.031239 & 6.315520 & 3.744679 \\
\hline $\mathrm{C}$ & -1.887297 & 6.220222 & 4.845401 \\
\hline $\mathrm{C}$ & -2.688866 & 5.087563 & 5.004309 \\
\hline $\mathrm{C}$ & -2.639327 & 4.052253 & 4.067961 \\
\hline $\mathrm{H}$ & -0.314123 & 5.352857 & 1.952835 \\
\hline $\mathrm{H}$ & -0.404181 & 7.193903 & 3.614635 \\
\hline $\mathrm{H}$ & -1.929563 & 7.025729 & 5.574068 \\
\hline $\mathrm{H}$ & -3.356061 & 5.007834 & 5.858481 \\
\hline $\mathrm{H}$ & -3.258809 & 3.169573 & 4.185542 \\
\hline $\mathrm{S}$ & 0.111460 & -0.430863 & 1.351628 \\
\hline $\mathrm{C}$ & -1.141834 & 0.709857 & 0.542798 \\
\hline $\mathrm{H}$ & -1.378038 & 0.216737 & -0.403742 \\
\hline
\end{tabular}


B3LYP/6-31G* Energy = -2089.06085977445

Number of Imaginary Frequencies $=1$

Imaginary Frequency $=-270.60$

TS1-RR

B3LYP/6-31G* GEOMETRY

\begin{tabular}{|c|c|c|c|}
\hline $\mathrm{C}$ & -0.001858 & -0.882890 & 0.634775 \\
\hline $\mathrm{C}$ & -0.164197 & -0.885915 & 2.102796 \\
\hline $\mathrm{C}$ & -1.333031 & -0.419198 & 2.743472 \\
\hline $\mathrm{C}$ & -1.481397 & -0.526805 & 4.125839 \\
\hline $\mathrm{C}$ & -0.483251 & -1.116374 & 4.906623 \\
\hline $\mathrm{C}$ & 0.671875 & -1.597965 & 4.284064 \\
\hline $\mathrm{C}$ & 0.834719 & -1.476301 & 2.905076 \\
\hline $\mathrm{C}$ & 0.757154 & 1.980282 & 0.082699 \\
\hline $\mathrm{C}$ & 0.633967 & 1.636171 & -2.380762 \\
\hline $\mathrm{C}$ & 1.555015 & 2.033305 & -1.235847 \\
\hline $\mathrm{O}$ & -0.146962 & 2.781101 & -2.679415 \\
\hline $\mathrm{O}$ & 2.630033 & 1.109421 & -1.237725 \\
\hline $\mathrm{C}$ & 1.682585 & 1.827836 & 1.303758 \\
\hline $\mathrm{O}$ & 2.765709 & 0.935308 & 1.065679 \\
\hline $\mathrm{C}$ & 3.464552 & 1.286834 & -0.102273 \\
\hline $\mathrm{C}$ & 4.713996 & 0.457255 & -0.278290 \\
\hline $\mathrm{C}$ & 5.320612 & 0.409422 & -1.540814 \\
\hline $\mathrm{C}$ & 6.513764 & -0.289175 & -1.720535 \\
\hline $\mathrm{C}$ & 7.110846 & -0.943534 & -0.639213 \\
\hline $\mathrm{C}$ & 6.508609 & -0.893964 & 0.620030 \\
\hline $\mathrm{C}$ & 5.313508 & -0.192897 & 0.804028 \\
\hline $\mathrm{C}$ & -1.512287 & 0.326307 & -2.798712 \\
\hline $\mathrm{O}$ & -2.103438 & 1.598819 & -3.048200 \\
\hline $\mathrm{C}$ & -1.178258 & 2.479899 & -3.617584 \\
\hline $\mathrm{C}$ & -1.832099 & 3.765836 & -4.063111 \\
\hline $\mathrm{C}$ & -1.032737 & 4.887194 & -4.319863 \\
\hline $\mathrm{C}$ & -1.604842 & 6.058650 & -4.815123 \\
\hline $\mathrm{C}$ & -2.979895 & 6.116887 & -5.059686 \\
\hline $\mathrm{C}$ & -3.777669 & 4.999379 & -4.803723 \\
\hline $\mathrm{C}$ & -3.207256 & 3.824060 & -4.308145 \\
\hline S & -0.572077 & 0.658641 & -0.118601 \\
\hline $\mathrm{C}$ & -0.236697 & 0.439767 & -1.949355 \\
\hline $\mathrm{H}$ & 1.017681 & -1.091675 & 0.306785 \\
\hline $\mathrm{H}$ & -2.137874 & 0.001205 & 2.146969 \\
\hline $\mathrm{H}$ & -2.391466 & -0.158942 & 4.594069 \\
\hline $\mathrm{H}$ & -0.605308 & -1.202864 & 5.983197 \\
\hline $\mathrm{H}$ & 1.455721 & -2.065012 & 4.876081 \\
\hline $\mathrm{H}$ & 1.744307 & -1.841137 & 2.434054 \\
\hline $\mathrm{H}$ & 1.233871 & 1.341082 & -3.255890 \\
\hline $\mathrm{H}$ & 0.177167 & 2.902785 & 0.184012 \\
\hline $\mathrm{H}$ & 1.930981 & 3.055510 & -1.398958 \\
\hline $\mathrm{H}$ & 2.078901 & 2.827902 & 1.545367 \\
\hline $\mathrm{H}$ & 1.151350 & 1.443244 & 2.174935 \\
\hline $\mathrm{H}$ & 3.727101 & 2.362144 & -0.044581 \\
\hline $\mathrm{H}$ & 4.850876 & 0.914306 & -2.379343 \\
\hline $\mathrm{H}$ & 6.975795 & -0.322852 & -2.703920 \\
\hline $\mathrm{H}$ & 8.040916 & -1.488366 & -0.779154 \\
\hline $\mathrm{H}$ & 6.969148 & -1.401798 & 1.463205 \\
\hline $\mathrm{H}$ & 4.842415 & -0.150615 & 1.780496 \\
\hline $\mathrm{H}$ & -1.230287 & -0.142649 & -3.756100 \\
\hline $\mathrm{H}$ & -2.243426 & -0.297243 & -2.277028 \\
\hline $\mathrm{H}$ & -0.698401 & 1.980545 & -4.483045 \\
\hline $\mathrm{H}$ & 0.034601 & 4.838506 & -4.126410 \\
\hline $\mathrm{H}$ & -0.977290 & 6.924321 & -5.011331 \\
\hline $\mathrm{H}$ & -3.425525 & 7.028671 & -5.449110 \\
\hline $\mathrm{H}$ & -4.847403 & 5.039951 & -4.991298 \\
\hline $\mathrm{H}$ & -3.821156 & 2.952196 & -4.108401 \\
\hline $\mathrm{H}$ & 0.340425 & -0.485872 & -2.017641 \\
\hline $\mathrm{C}$ & -1.405364 & -2.065956 & -0.301500 \\
\hline $\mathrm{O}$ & -2.392286 & -1.301158 & -0.544754 \\
\hline $\mathrm{C}$ & -1.588661 & -3.226751 & 0.640252 \\
\hline $\mathrm{C}$ & -2.798706 & -3.339063 & 1.336847 \\
\hline $\mathrm{C}$ & -3.030930 & -4.423224 & 2.183096 \\
\hline $\mathrm{C}$ & -2.052402 & -5.407904 & 2.345090 \\
\hline $\mathrm{C}$ & -0.842199 & -5.304387 & 1.651140 \\
\hline $\mathrm{C}$ & -0.614738 & -4.220818 & 0.800737 \\
\hline $\mathrm{H}$ & -0.691501 & -2.321503 & -1.117612 \\
\hline $\mathrm{H}$ & -3.547027 & -2.569148 & 1.177039 \\
\hline $\mathrm{H}$ & -3.977039 & -4.503065 & 2.713970 \\
\hline $\mathrm{H}$ & -2.232767 & -6.253476 & 3.004872 \\
\hline
\end{tabular}

$\begin{array}{lrrr}\mathrm{H} & -0.080083 & -6.070843 & 1.771949 \\ \mathrm{H} & 0.325360 & -4.146058 & 0.255541\end{array}$

B3LYP/6-31G* Energy $=-2089.06392547304$

Number of Imaginary Frequencies $=1$

Imaginary Frequency $=-252.20$

TS1-SS

B3LYP/6-31G* GEOMETRY

\begin{tabular}{|c|c|c|c|}
\hline $\mathrm{C}$ & 0.165227 & -1.151673 & 1.246283 \\
\hline $\mathrm{C}$ & 0.388459 & -1.898011 & -0.014196 \\
\hline $\mathrm{C}$ & -0.641087 & -2.055313 & -0.966264 \\
\hline $\mathrm{C}$ & -0.455565 & -2.850963 & -2.097370 \\
\hline $\mathrm{C}$ & 0.744996 & -3.536980 & -2.290680 \\
\hline $\mathrm{C}$ & 1.758615 & -3.426511 & -1.335057 \\
\hline $\mathrm{C}$ & 1.584990 & -2.612275 & -0.217518 \\
\hline $\mathrm{H}$ & 0.957612 & -1.314520 & 1.985126 \\
\hline $\mathrm{H}$ & -1.610427 & -1.607418 & -0.769807 \\
\hline $\mathrm{H}$ & -1.264266 & -2.956336 & -2.816543 \\
\hline $\mathrm{H}$ & 0.885665 & -4.162083 & -3.168622 \\
\hline $\mathrm{H}$ & 2.691651 & -3.969491 & -1.464871 \\
\hline $\mathrm{H}$ & 2.386090 & -2.524275 & 0.512170 \\
\hline $\mathrm{C}$ & -1.572326 & -1.553063 & 2.190629 \\
\hline $\mathrm{O}$ & -2.489975 & -0.879015 & 1.630123 \\
\hline $\mathrm{C}$ & -1.651695 & -3.065641 & 2.139562 \\
\hline $\mathrm{C}$ & -0.808999 & -3.888386 & 2.896878 \\
\hline $\mathrm{C}$ & -0.946437 & -5.277581 & 2.854332 \\
\hline $\mathrm{C}$ & -1.934916 & -5.859759 & 2.053669 \\
\hline $\mathrm{C}$ & -2.787087 & -5.043958 & 1.304217 \\
\hline $\mathrm{C}$ & -2.646439 & -3.656856 & 1.351110 \\
\hline $\mathrm{H}$ & -1.159688 & -1.203649 & 3.165031 \\
\hline $\mathrm{H}$ & -0.044361 & -3.438422 & 3.529323 \\
\hline $\mathrm{H}$ & -0.288087 & -5.908301 & 3.447605 \\
\hline $\mathrm{H}$ & -2.044725 & -6.941402 & 2.020556 \\
\hline $\mathrm{H}$ & -3.564899 & -5.490205 & 0.687961 \\
\hline $\mathrm{H}$ & -3.311254 & -2.998361 & 0.800673 \\
\hline $\mathrm{C}$ & 1.517016 & 1.646937 & 0.754444 \\
\hline $\mathrm{C}$ & -0.247343 & 2.428614 & -0.810303 \\
\hline $\mathrm{C}$ & 1.252706 & 2.238753 & -0.642067 \\
\hline $\mathrm{H}$ & -0.492400 & 2.523036 & -1.879931 \\
\hline $\mathrm{O}$ & -0.580096 & 3.621246 & -0.118854 \\
\hline $\mathrm{O}$ & 1.681696 & 1.371365 & -1.675054 \\
\hline $\mathrm{H}$ & 1.513808 & 2.456072 & 1.490924 \\
\hline $\mathrm{H}$ & 1.758408 & 3.213560 & -0.728770 \\
\hline $\mathrm{C}$ & 2.863079 & 0.909134 & 0.824750 \\
\hline $\mathrm{H}$ & 3.644239 & 1.665193 & 1.010150 \\
\hline $\mathrm{H}$ & 2.888783 & 0.187418 & 1.645424 \\
\hline $\mathrm{O}$ & 3.172530 & 0.185791 & -0.356921 \\
\hline $\mathrm{C}$ & 3.037656 & 0.995450 & -1.500771 \\
\hline $\mathrm{H}$ & 3.623906 & 1.922932 & -1.340346 \\
\hline $\mathrm{C}$ & 3.521484 & 0.292569 & -2.746728 \\
\hline $\mathrm{C}$ & 3.140102 & 0.799886 & -3.995871 \\
\hline $\mathrm{C}$ & 3.634325 & 0.227889 & -5.167592 \\
\hline $\mathrm{C}$ & 4.515472 & -0.854853 & -5.099633 \\
\hline $\mathrm{C}$ & 4.895638 & -1.361527 & -3.854981 \\
\hline $\mathrm{C}$ & 4.403318 & -0.789197 & -2.678994 \\
\hline $\mathrm{H}$ & 2.449925 & 1.636468 & -4.045107 \\
\hline $\mathrm{H}$ & 3.330567 & 0.626985 & -6.132066 \\
\hline $\mathrm{H}$ & 4.902340 & -1.300161 & -6.012728 \\
\hline $\mathrm{H}$ & 5.577476 & -2.205911 & -3.797561 \\
\hline $\mathrm{H}$ & 4.693735 & -1.182114 & -1.710654 \\
\hline $\mathrm{C}$ & -2.463733 & 1.538974 & 0.052382 \\
\hline $\mathrm{H}$ & -3.000739 & 1.534451 & -0.911099 \\
\hline $\mathrm{H}$ & -2.887930 & 0.793616 & 0.724611 \\
\hline $\mathrm{O}$ & -2.603725 & 2.815365 & 0.672039 \\
\hline $\mathrm{C}$ & -1.992914 & 3.820798 & -0.081836 \\
\hline $\mathrm{H}$ & -2.358395 & 3.756171 & -1.126331 \\
\hline $\mathrm{C}$ & -2.285937 & 5.189220 & 0.484936 \\
\hline $\mathrm{C}$ & -1.504462 & 6.279884 & 0.083883 \\
\hline $\mathrm{C}$ & -1.812401 & 7.565220 & 0.528723 \\
\hline $\mathrm{C}$ & -2.905183 & 7.769671 & 1.376070 \\
\hline $\mathrm{C}$ & -3.683692 & 6.682115 & 1.778245 \\
\hline $\mathrm{C}$ & -3.377901 & 5.393430 & 1.333671 \\
\hline $\mathrm{H}$ & -0.655054 & 6.116095 & -0.572454 \\
\hline $\mathrm{H}$ & -1.201208 & 8.406609 & 0.212233 \\
\hline $\mathrm{H}$ & -3.148722 & 8.772149 & 1.718722 \\
\hline $\mathrm{H}$ & -4.533522 & 6.835245 & 2.438305 \\
\hline
\end{tabular}




$\begin{array}{lllr}\mathrm{H} & -3.979107 & 4.544333 & 1.640989 \\ \mathrm{~S} & -0.029566 & 0.661330 & 1.263117 \\ \mathrm{C} & -0.994383 & 1.205131 & -0.246011 \\ \mathrm{H} & -0.918489 & 0.375073 & -0.951663\end{array}$

B3LYP/6-31G* Energy = -2089.05457981175

Number of Imaginary Frequencies $=1$

Imaginary Frequency $=-245.40$ 
Transition Structures for the Addition Reaction of Sulfonium Ylide 2 to Benzaldehyde.

TS2-RS

B3LYP/6-31G* GEOMETRY

\begin{tabular}{|c|c|c|c|}
\hline $\mathrm{C}$ & 0.786642 & -1.914719 & -0.967156 \\
\hline $\mathrm{C}$ & 0.699459 & -2.493569 & 0.405450 \\
\hline $\mathrm{C}$ & 1.705110 & -2.240245 & 1.363512 \\
\hline $\mathrm{C}$ & 1.650646 & -2.828871 & 2.627791 \\
\hline $\mathrm{C}$ & 0.613636 & -3.703766 & 2.961719 \\
\hline $\mathrm{C}$ & -0.359114 & -4.008243 & 2.005844 \\
\hline $\mathrm{C}$ & -0.314353 & -3.411393 & 0.745107 \\
\hline $\mathrm{H}$ & 0.177986 & -2.453650 & -1.698456 \\
\hline $\mathrm{H}$ & 2.559833 & -1.636988 & 1.066447 \\
\hline $\mathrm{H}$ & 2.437292 & -2.618460 & 3.348640 \\
\hline $\mathrm{H}$ & 0.575033 & -4.159863 & 3.947690 \\
\hline $\mathrm{H}$ & -1.154632 & -4.711383 & 2.240842 \\
\hline $\mathrm{H}$ & -1.068211 & -3.670327 & 0.004309 \\
\hline $\mathrm{C}$ & 2.657066 & -1.925909 & -1.591143 \\
\hline $\mathrm{O}$ & 3.312483 & -1.000345 & -1.015823 \\
\hline $\mathrm{C}$ & 2.481521 & -1.884633 & -3.105193 \\
\hline $\mathrm{C}$ & 2.916114 & -0.763619 & -3.820137 \\
\hline $\mathrm{C}$ & 2.764656 & -0.701007 & -5.207088 \\
\hline $\mathrm{C}$ & 2.184325 & -1.767125 & -5.899121 \\
\hline $\mathrm{C}$ & 1.766382 & -2.901683 & -5.195237 \\
\hline $\mathrm{C}$ & 1.918690 & -2.957837 & -3.809520 \\
\hline $\mathrm{H}$ & 2.792355 & -2.976978 & -1.245892 \\
\hline $\mathrm{H}$ & 3.386598 & 0.042160 & -3.264708 \\
\hline $\mathrm{H}$ & 3.106448 & 0.177295 & -5.750663 \\
\hline $\mathrm{H}$ & 2.066762 & -1.720462 & -6.979180 \\
\hline $\mathrm{H}$ & 1.327052 & -3.742025 & -5.728244 \\
\hline $\mathrm{H}$ & 1.602544 & -3.846579 & -3.264006 \\
\hline $\mathrm{C}$ & -1.420691 & 0.217462 & -0.648827 \\
\hline $\mathrm{C}$ & -0.018513 & 1.543639 & 0.849309 \\
\hline $\mathrm{C}$ & -1.246396 & 0.630914 & 0.813240 \\
\hline $\mathrm{H}$ & 0.309560 & 1.755144 & 1.873358 \\
\hline $\mathrm{H}$ & -0.259900 & 2.507066 & 0.380194 \\
\hline $\mathrm{H}$ & -1.088642 & -0.258982 & 1.433579 \\
\hline $\mathrm{H}$ & -1.667252 & 1.102823 & -1.250096 \\
\hline $\mathrm{H}$ & -2.147381 & 1.140443 & 1.177482 \\
\hline $\mathrm{S}$ & 0.323531 & -0.185569 & -1.254079 \\
\hline $\mathrm{C}$ & 1.135657 & 0.870160 & 0.080619 \\
\hline $\mathrm{H}$ & 1.648026 & 0.147026 & 0.712760 \\
\hline $\mathrm{C}$ & -2.428306 & -0.887377 & -0.934617 \\
\hline $\mathrm{H}$ & -2.218703 & -1.778706 & -0.339687 \\
\hline $\mathrm{H}$ & -3.431197 & -0.529866 & -0.672485 \\
\hline $\mathrm{H}$ & -2.439490 & -1.165155 & -1.993872 \\
\hline $\mathrm{C}$ & 2.145484 & 1.833840 & -0.531747 \\
\hline $\mathrm{H}$ & 2.944874 & 1.263299 & -1.008334 \\
\hline $\mathrm{H}$ & 1.672715 & 2.507361 & -1.256392 \\
\hline $\mathrm{H}$ & 2.573636 & 2.447743 & 0.270726 \\
\hline
\end{tabular}

B3LYP/6-31G* Energy = -1249.90440387331

Number of Imaginary Frequencies $=1$

Imaginary Frequency $=-220.05$

TS2-SR

B3LYP/6-31G* GEOMETRY

$\begin{array}{lrrr}\mathrm{C} & 0.076260 & -1.915227 & 0.301828 \\ \mathrm{C} & 1.227173 & -2.828782 & 0.418923 \\ \mathrm{C} & 1.721874 & -3.263103 & 1.667900 \\ \mathrm{C} & 2.744258 & -4.207610 & 1.744113 \\ \mathrm{C} & 3.286711 & -4.765360 & 0.582506 \\ \mathrm{C} & 2.791805 & -4.363226 & -0.661267 \\ \mathrm{C} & 1.783115 & -3.403895 & -0.743045 \\ \mathrm{H} & -0.106339 & -1.570662 & -0.717133 \\ \mathrm{H} & 1.288723 & -2.865588 & 2.582887 \\ \mathrm{H} & 3.109979 & -4.520078 & 2.719366 \\ \mathrm{H} & 4.079311 & -5.505905 & 0.646088 \\ \mathrm{H} & 3.198927 & -4.790993 & -1.574491 \\ \mathrm{H} & 1.411538 & -3.092476 & -1.717327 \\ \mathrm{C} & -1.563481 & -2.753576 & 1.088231 \\ \mathrm{O} & -1.986703 & -1.978727 & 2.008474 \\ \mathrm{C} & -2.420265 & -2.995489 & -0.143196 \\ \mathrm{C} & -2.049824 & -3.928902 & -1.122561 \\ \mathrm{C} & -2.850259 & -4.135765 & -2.246335\end{array}$

$\begin{array}{lrrr}\mathrm{C} & -4.041379 & -3.417633 & -2.401978 \\ \mathrm{C} & -4.427936 & -2.501501 & -1.420342 \\ \mathrm{C} & -3.623219 & -2.296113 & -0.296983 \\ \mathrm{H} & -1.014792 & -3.675548 & 1.373914 \\ \mathrm{H} & -1.127488 & -4.493553 & -0.998904 \\ \mathrm{H} & -2.550659 & -4.860725 & -3.000005 \\ \mathrm{H} & -4.667428 & -3.581020 & -3.276039 \\ \mathrm{H} & -5.363170 & -1.955422 & -1.525511 \\ \mathrm{H} & -3.918584 & -1.614005 & 0.495611 \\ \mathrm{C} & 1.385953 & 0.710663 & 0.725551 \\ \mathrm{C} & -0.719475 & 1.723494 & -0.011330 \\ \mathrm{C} & 0.692539 & 1.356791 & -0.475603 \\ \mathrm{H} & -1.381972 & 1.960597 & -0.851320 \\ \mathrm{H} & -0.671106 & 2.617839 & 0.623260 \\ \mathrm{H} & 0.663574 & 0.645419 & -1.310284 \\ \mathrm{H} & 1.466474 & 1.452488 & 1.532455 \\ \mathrm{H} & 1.252949 & 2.236578 & -0.814934 \\ \mathrm{~S} & 0.117615 & -0.488798 & 1.420977 \\ \mathrm{C} & -1.324566 & 0.557700 & 0.804891 \\ \mathrm{H} & -1.875370 & -0.137200 & 0.169776 \\ \mathrm{C} & 2.759231 & 0.106703 & 0.456217 \\ \mathrm{H} & 2.724167 & -0.628181 & -0.351225 \\ \mathrm{H} & 3.443918 & 0.911863 & 0.163497 \\ \mathrm{H} & 3.172537 & -0.385633 & 1.341287 \\ \mathrm{C} & -2.205781 & 1.010936 & 1.963941 \\ \mathrm{H} & -2.624340 & 0.140263 & 2.468897 \\ \mathrm{H} & -1.645246 & 1.626534 & 2.677843 \\ \mathrm{H} & -3.020112 & 1.626929 & 1.561227\end{array}$

B3LYP/6-31G* Energy $=-1249.90715088421$

Number of Imaginary Frequencies $=1$

Imaginary Frequency $=-255.95$

TS2-RR

B3LYP/6-31G* GEOMETRY

\begin{tabular}{|c|c|c|c|}
\hline $\mathrm{C}$ & -0.107062 & -0.852644 & 0.622155 \\
\hline $\mathrm{C}$ & -0.242458 & -0.843659 & 2.093942 \\
\hline $\mathrm{C}$ & -1.385237 & -0.331502 & 2.744053 \\
\hline $\mathrm{C}$ & -1.507876 & -0.401360 & 4.131650 \\
\hline $\mathrm{C}$ & -0.509402 & -0.996488 & 4.906974 \\
\hline $\mathrm{C}$ & 0.615700 & -1.531813 & 4.273619 \\
\hline $\mathrm{C}$ & 0.749978 & -1.451445 & 2.888935 \\
\hline $\mathrm{C}$ & 0.775170 & 1.951376 & 0.051705 \\
\hline $\mathrm{C}$ & 0.792919 & 1.458539 & -2.349136 \\
\hline $\mathrm{C}$ & 1.700697 & 1.678660 & -1.136796 \\
\hline $\mathrm{H}$ & 0.328873 & 2.409536 & -2.642517 \\
\hline $\mathrm{H}$ & 2.304759 & 0.783663 & -0.936535 \\
\hline $\mathrm{S}$ & -0.630920 & 0.708239 & -0.129479 \\
\hline $\mathrm{C}$ & -0.302958 & 0.448803 & -1.966402 \\
\hline $\mathrm{H}$ & 0.896113 & -1.115964 & 0.275266 \\
\hline $\mathrm{H}$ & -2.189868 & 0.093593 & 2.151026 \\
\hline $\mathrm{H}$ & -2.397699 & 0.002882 & 4.608726 \\
\hline $\mathrm{H}$ & -0.608926 & -1.048621 & 5.987956 \\
\hline $\mathrm{H}$ & 1.397189 & -2.008680 & 4.860694 \\
\hline $\mathrm{H}$ & 1.632954 & -1.868575 & 2.408467 \\
\hline $\mathrm{H}$ & 1.343107 & 1.084968 & -3.220370 \\
\hline $\mathrm{H}$ & 0.261304 & 2.908541 & -0.108012 \\
\hline $\mathrm{H}$ & 2.393131 & 2.516245 & -1.284150 \\
\hline $\mathrm{H}$ & 0.092570 & -0.569543 & -2.009991 \\
\hline $\mathrm{C}$ & -1.504502 & -1.986703 & -0.268419 \\
\hline $\mathrm{O}$ & -2.518415 & -1.235283 & -0.430670 \\
\hline $\mathrm{C}$ & -1.589827 & -3.195286 & 0.631572 \\
\hline $\mathrm{C}$ & -2.695970 & -3.348270 & 1.474860 \\
\hline $\mathrm{C}$ & -2.805214 & -4.469119 & 2.298712 \\
\hline $\mathrm{C}$ & -1.810560 & -5.451267 & 2.285205 \\
\hline $\mathrm{C}$ & -0.706883 & -5.309271 & 1.438247 \\
\hline $\mathrm{C}$ & -0.601585 & -4.187137 & 0.615625 \\
\hline $\mathrm{H}$ & -0.851502 & -2.217491 & -1.144515 \\
\hline $\mathrm{H}$ & -3.462158 & -2.579459 & 1.451813 \\
\hline $\mathrm{H}$ & -3.668468 & -4.580379 & 2.951122 \\
\hline $\mathrm{H}$ & -1.896526 & -6.325283 & 2.926651 \\
\hline $\mathrm{H}$ & 0.065663 & -6.074730 & 1.417149 \\
\hline $\mathrm{H}$ & 0.253564 & -4.079269 & -0.051290 \\
\hline $\mathrm{C}$ & 1.435129 & 1.952443 & 1.425150 \\
\hline $\mathrm{H}$ & 1.948402 & 1.008587 & 1.624980 \\
\hline $\mathrm{H}$ & 2.173705 & 2.762479 & 1.459467 \\
\hline $\mathrm{H}$ & 0.710666 & 2.115163 & 2.227874 \\
\hline
\end{tabular}




$\begin{array}{lrrr}\mathrm{C} & -1.593260 & 0.543231 & -2.773139 \\ \mathrm{H} & -2.329610 & -0.155588 & -2.366009 \\ \mathrm{H} & -2.003221 & 1.559660 & -2.742764 \\ \mathrm{H} & -1.378832 & 0.294040 & -3.819665\end{array}$

B3LYP/6-31G* Energy $=-1249.90556450733$

Number of Imaginary Frequencies $=1$

Imaginary Frequency $=-255.62$

\section{TS2-SS}

B3LYP/6-31G* GEOMETRY

$\begin{array}{lrrr}\mathrm{C} & 0.031552 & -1.160517 & 1.277000 \\ \mathrm{C} & 0.381846 & -1.857221 & 0.005844 \\ \mathrm{C} & -0.518377 & -1.933657 & -1.077196 \\ \mathrm{C} & -0.183658 & -2.632751 & -2.237909 \\ \mathrm{C} & 1.041289 & -3.296544 & -2.341317 \\ \mathrm{C} & 1.921562 & -3.276747 & -1.256569 \\ \mathrm{C} & 1.594256 & -2.566999 & -0.100991 \\ \mathrm{H} & 0.726358 & -1.401087 & 2.087161 \\ \mathrm{H} & -1.508684 & -1.506139 & -0.965678 \\ \mathrm{H} & -0.896629 & -2.678169 & -3.057885 \\ \mathrm{H} & 1.297995 & -3.839868 & -3.247136 \\ \mathrm{H} & 2.865784 & -3.813476 & -1.308904 \\ \mathrm{H} & 2.283797 & -2.568113 & 0.740917 \\ \mathrm{C} & -1.741041 & -1.547097 & 1.932084 \\ \mathrm{O} & -2.611703 & -0.966914 & 1.199890 \\ \mathrm{C} & -1.721497 & -3.070500 & 1.977949 \\ \mathrm{C} & -0.943482 & -3.778320 & 2.902242 \\ \mathrm{C} & -0.958103 & -5.173872 & 2.925032 \\ \mathrm{C} & -1.761754 & -5.878902 & 2.023259 \\ \mathrm{C} & -2.555184 & -5.178374 & 1.110647 \\ \mathrm{C} & -2.536650 & -3.782845 & 1.092199 \\ \mathrm{H} & -1.547102 & -1.142310 & 2.955301 \\ \mathrm{H} & -0.325286 & -3.231330 & 3.613900 \\ \mathrm{H} & -0.347684 & -5.712744 & 3.646363 \\ \mathrm{H} & -1.774833 & -6.966292 & 2.038478 \\ \mathrm{H} & -3.191689 & -5.721693 & 0.415341 \\ \mathrm{H} & -3.158518 & -3.211458 & 0.409722 \\ \mathrm{C} & 1.482948 & 1.443626 & 0.477294 \\ \mathrm{C} & -0.361260 & 2.099020 & -0.992800 \\ \mathrm{C} & 1.076462 & 1.571823 & -0.992334 \\ \mathrm{H} & -0.819695 & 2.036912 & -1.986291 \\ \mathrm{H} & -0.366141 & 3.157308 & -0.698971 \\ \mathrm{H} & 1.131840 & 0.591498 & -1.478523 \\ \mathrm{H} & 1.499797 & 2.441289 & 0.936469 \\ \mathrm{H} & 1.760203 & 2.249431 & -1.518908 \\ \mathrm{~S} & -0.007576 & 0.656817 & 1.322341 \\ \mathrm{C} & -1.209128 & 1.283801 & 0.004384 \\ \mathrm{H} & -1.602157 & 0.373687 & -0.439535 \\ \mathrm{C} & 2.794495 & 0.726326 & 0.767451 \\ \mathrm{H} & 2.813658 & -0.266196 & 0.312752 \\ \mathrm{H} & 3.618922 & 1.313174 & 0.345395 \\ \mathrm{H} & 2.974067 & 0.625648 & 1.843167 \\ \mathrm{C} & -2.353118 & 2.049132 & 0.658427 \\ \mathrm{H} & -2.919949 & 1.364434 & 1.292771 \\ \mathrm{H} & -1.994629 & 2.908763 & 1.237165 \\ \mathrm{H} & -3.015952 & 2.425588 & -0.131057\end{array}$

B3LYP/6-31G* Energy $=-1249.90374040230$

Number of Imaginary Frequencies $=1$

Imaginary Frequency $=-213.12$ 
Single Point Energies in Acetonitrile for the Transition Structures for the Addition Reaction (trans pathway) of Sulfonium Ylide 2 to Benzaldehyde (in hartrees).

\section{TS2-RR}

B3LYP/6-31G*(acetonitrile)//B3LYP/6-31G* $=-1249.96158008981$
TS2-SS

B3LYP/6-31G*(acetonitrile)//B3LYP/6-31G* $=-1249.9561175819$

Figure S1. QRC Calculations at the B3LYP/6-31G* level for the TS2s. Plots of the relative energy (in $\mathrm{kcal}^{\mathrm{mol}}{ }^{-1}$ ) versus mass-weighted coordinates (in bohr amu ${ }^{1 / 2}$ ).
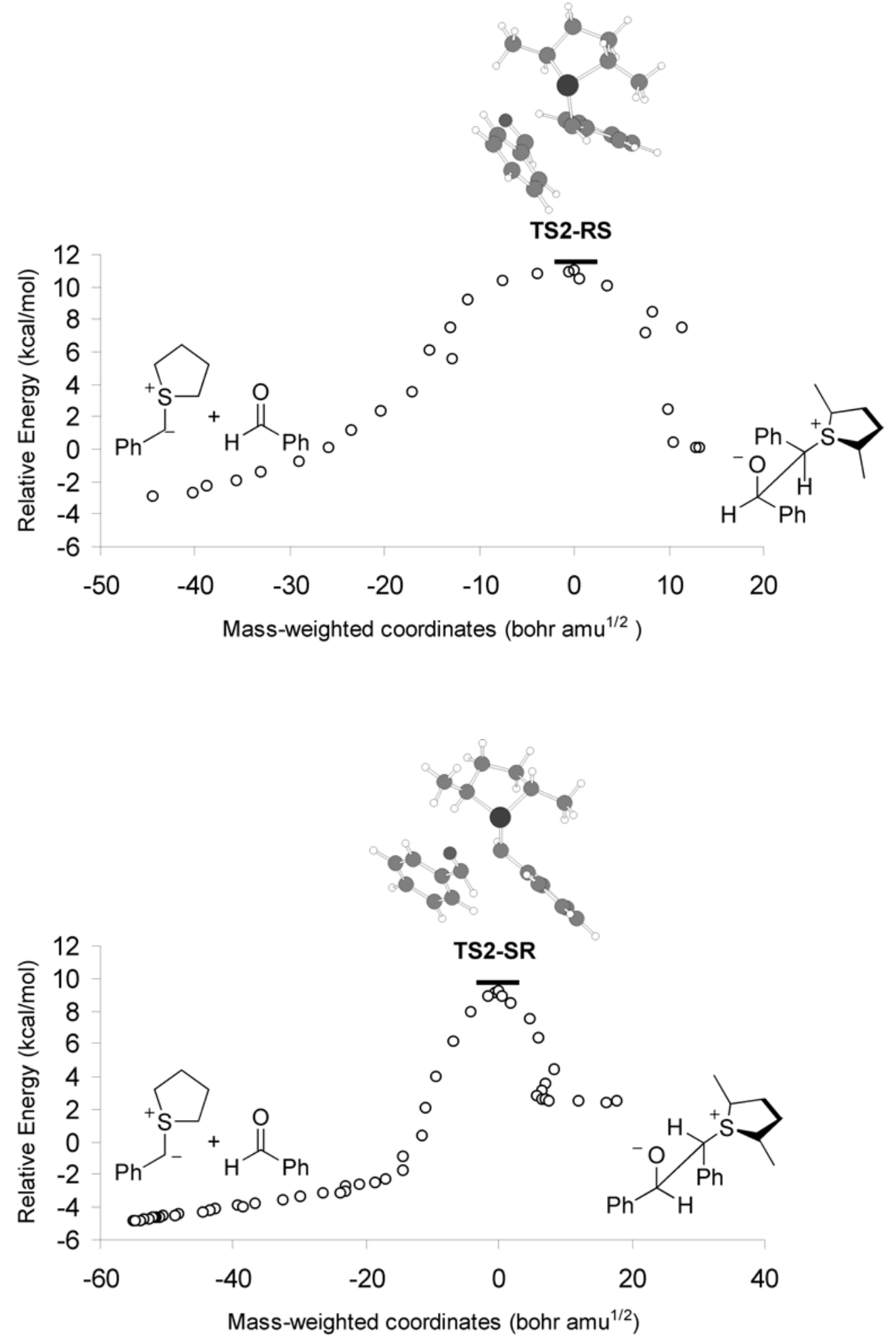

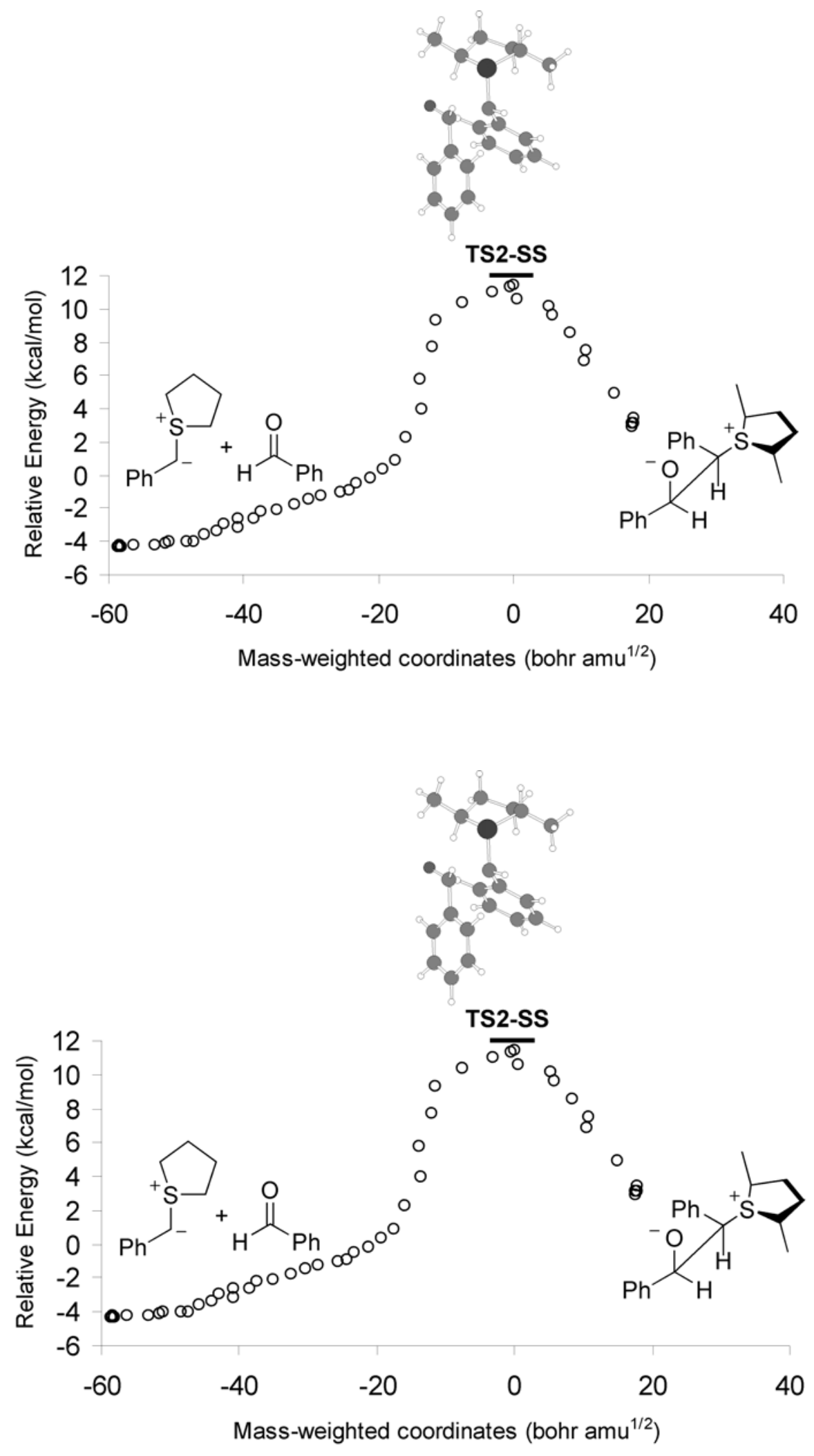
Figure S2. B3LYP/6-31G* Geometries for the elimination (betaines to epoxides) transition structures with sulfide 2.

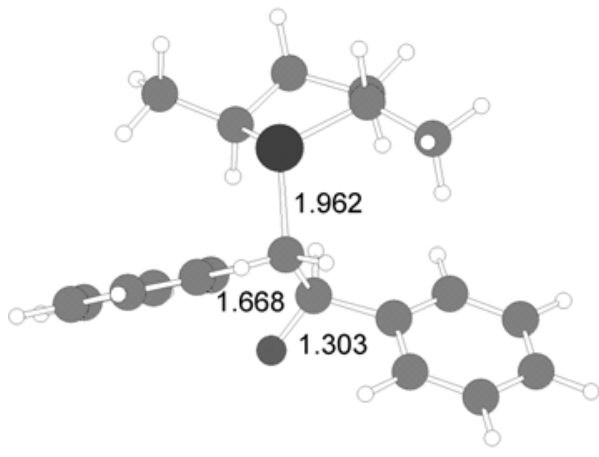

trans-RR

B3LYP/6-31G* Energy $=-1249.88340993559$

Number of Imaginary Frequencies $=1$

B3LYP/6-31G*(acetonitrile)//B3LYP/6-31G* = -1249.97077557614

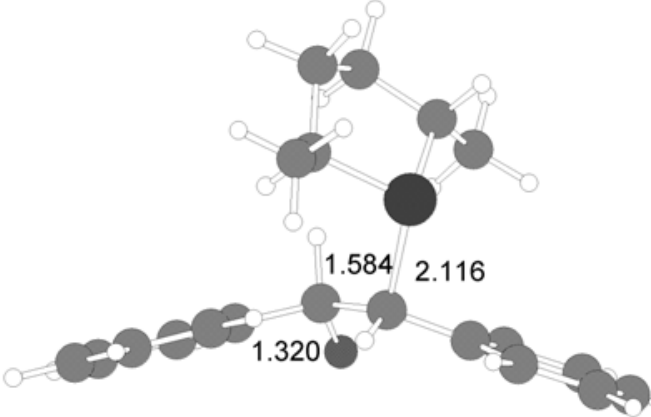

trans-SS

B3LYP/6-31G* Energy $=-1249.87779873438$

Number of Imaginary Frequencies $=1$

B3LYP/6-31G*(acetonitrile)//B3LYP/6-31G* = -1249.95734643665 
Figure S3. QRC calculations at the B3LYP/6-31G* level for the TSs connecting betaines with epoxides for the trans pathway and with the chiral sulfide 2. Plots of the relative energy (in $\mathrm{kcal} \mathrm{mol}^{-1}$, relative to the reactants) versus mass-weighted coordinates (in bohr amu ${ }^{1 / 2}$ ).
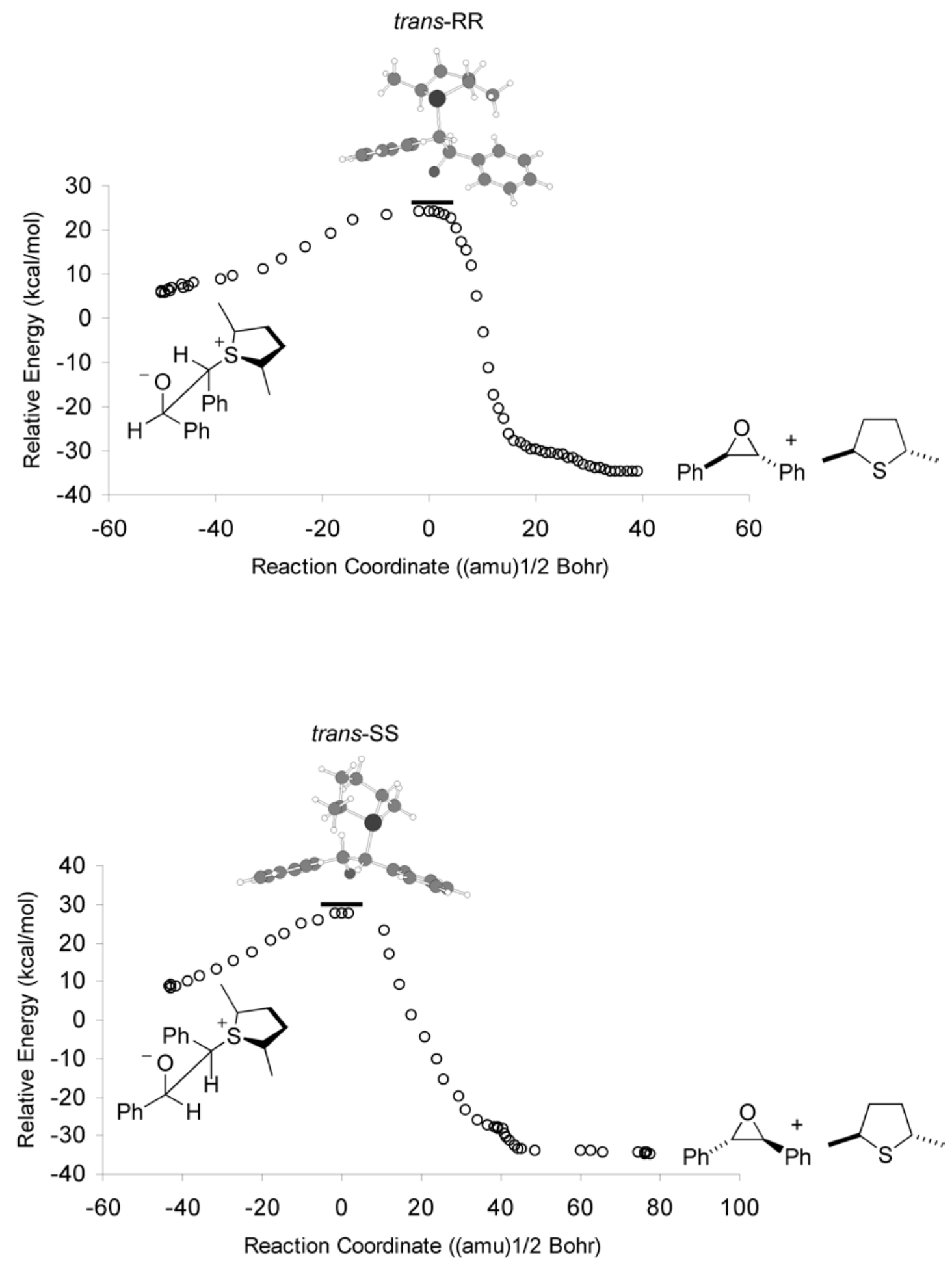